\title{
Radiography Calibration Marker Detection using Hough Transformation
}

\author{
Lena D. Novović, Vladimir S. Ostojić, Đorđe S. Starčević, and Vladimir S. Petrović
}

\begin{abstract}
In this paper we analyse the possibility of simple detection of circular radiography markers. To detect the marker, we utilised the Hough transform. Two approaches were analysed: with detecting image edges and without image edge detection where pixel gradient was used in the Hough voting process, i.e. to increase the accumulator values. Approaches were evaluated on 13 clinical radiography images. It was shown that the approach that detects image edges spatially matches the reference circles only $0.22 \%$ less than manual annotation values, whereas the approach that uses just the gradient magnitudes spatially matches the reference circles $3.2 \%$ less than manual annotations.
\end{abstract}

Keywords - digital radiography, image delineation, magnification detection, radiography marker.

\section{INTRODUCTION}

$\mathrm{B}$ LOCK diagram representing a radiography imaging system is shown in Fig.1. As can be seen, patient is an absorber positioned between the radiation source and the detector [1]. X-ray beam attenuates as it progresses through the body, with the attenuation being more prominent in the tissues with higher thickness. A collimator is used to limit the radiation beam to the body part of interest, while the anti-scatter grid is used to remove the scattered radiation [2].

Radiography image pixel intensities are linearly proportional to the intensity of the beam at the surface of the detector [3]. Since pixel intensities are affected by the attenuation of the beam, the radiography image represents the projection of the imaged anatomy. If the x-ray source is considered a point source, the projection of the anatomy

Paper received March 19, 2019; revised April 04, 2019; accepted April 17, 2019. Date of publication July 31, 2019. The associate editor coordinating the review of this manuscript and approving it for publication was Prof. Branimir Reljin.

This paper is revised and expanded version of the paper presented at the 26th Telecommunications Forum TELFOR 2018 [7].

This research is supported by grants TR32035 and TR32040 of Serbian Ministry of Science.

Lena D. Novović is a student at the University of Novi Sad, Serbia, Faculty of Technical Sciences, Biomedical engineering (e-mail: novovic.lena@gmail.com).

Vladimir S. Ostojic is with the University of Novi Sad, Serbia, Faculty of Technical Sciences, Department of Power, Electronics and Telecommunications (e-mail: vladimir.ostojic@uns.ac.rs).

Đorđe S. Starčević is with the University of Novi Sad, Serbia, Faculty of Technical Sciences, Department of Power, Electronics and Telecommunications (e-mail: djordje.starcevic@uns.ac.rs).

Vladimir S. Petrović is with the University of Novi Sad, Serbia, Faculty of Technical Sciences, Department of Power, Electronics and Telecommunications (e-mail: vladimir.petrovic@uns.ac.rs). will be larger than the anatomy itself, as can be deduced from Fig. 1.

Magnification factor depends on the positioning of the imaging system and the patient. As it is impractical to measure all the relevant positions in the system for every patient examination, calibration markers are used [4]. Markers are usually made from a radiopaque material [5] and are positioned near the anatomy of interest so that the magnification of their projection is approximately the same as of the anatomy. An example of radiography image with a calibration marker is shown in Fig. 2.

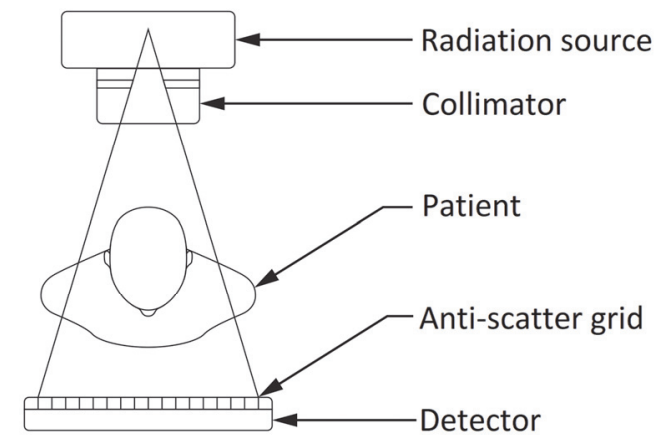

Fig. 1. Radiography imaging system diagram.

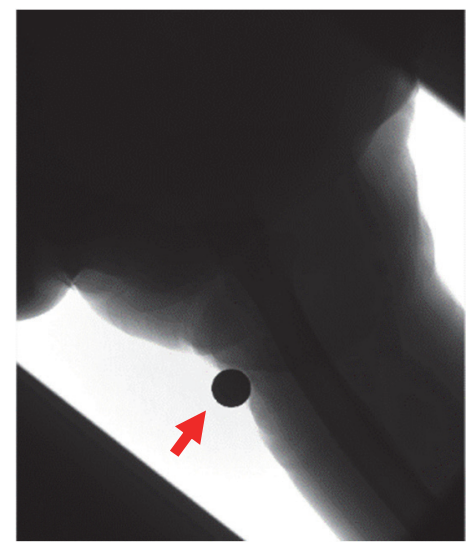

Fig. 2. Example of raw image with radiography calibration marker indicated by arrow.

Measuring the size of the marker projection can be used to determine the magnification inherently caused by the imaging system, as the calibration marker size is a priori known. This task requires manual selection of the marker and requires time and precision. In this paper we analysed the possibility of automatic calibration marker detection. Similar to [4], we analysed the possibility of using the Hough transform [6] for circular marker detection. In contrast to [4], we did not use least-square error fitting procedure. We simply used the circle that had the most votes. Additionally, we analysed the use of the gradient 
image as the basis for the Hough transform voting. Preliminary results are presented in [7], while this paper presents an expanded explanation of analysed approaches.

Section II presents the method. In Section III we outline the evaluation and present the obtained results. Section IV concludes the paper.

\section{METHOD}

\section{A. The Hough transform}

The Hough transform [6] is an image processing technique used to find objects that belong to a certain class of shapes. The technique uses a voting procedure that is done in a parameter space. Object candidates are determined as local maxima in the parameter space after voting.

Since the used calibration markers have a circular shape, we used the circular object detection variant of the Hough transform. The equation of a circle is:

$$
r^{2}=(X-x)^{2}+(Y-y)^{2}
$$

where $r$ is the radius of the circle, $x$ and $y$ are the spatial coordinates, while $X$ and $Y$ are the coordinates of the circle centre. It can be observed that the calibration markers are defined by their centre coordinates and radius. These three parameters define the three-dimensional Hough parameter space.

Votes of a pixel (in the image) will be distributed circularly in the parameter space. The circle centre coordinates will be the same as the ones of the analysed pixel, and the radii will increase along with the radius parameter in the parameter space. Therefore, every analysed pixel will produce a cone in the parameter space $(x, y, r)$, see Fig. 3 . To reduce the computational burden, we used a priori knowledge to limit the values of the radii parameter $r$ to the range [Rmin, Rmax].

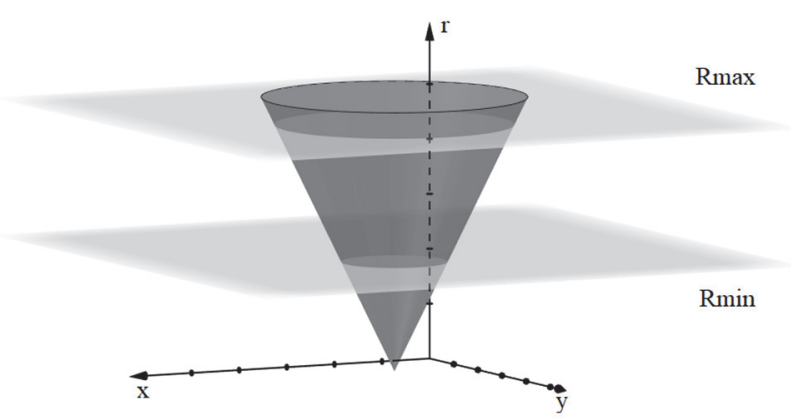

Fig. 3. Circular Hough transform of an image pixel.

Pixels that lie on a circle defined by the parameters $\left(x_{0}, y_{0}, r_{0}\right)$ will produce intersecting cones in the parameter space. The intersection point of these cones will be the point $\left(x_{0}, y_{0}, r_{0}\right)$. This is illustrated in Fig. 4. It shows the circular Hough transform of multiple pixels which belong to the same circle (the circle is illustrated in red). The intersection of the cones is shown in the plane $r=r_{0}$. The circular Hough transform of the pixels B, C, D and E is illustrated with grey circles. The point $\mathrm{A}$ is the point of intersection of the Hough transform circles, and the centre of the original circle.
The most discernible circle in an image will produce the biggest number of cones intersecting in the parameter space.

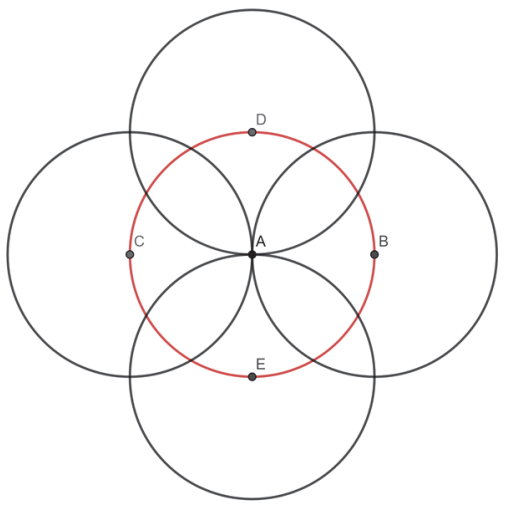

Fig. 4. 2D illustration of circular Hough transform of pixels $\mathrm{B}, \mathrm{C}, \mathrm{D}$ and $\mathrm{E}$. Centre of the circle is marked as point A.

With the purpose of detecting circle centre and radius detection, a three-dimensional accumulation matrix is used to find the intersection points in the parameter space.

Pixels casted votes to the accumulators that correspond to different circle centres and radii, and the cell with the biggest value in the accumulation matrix defines the point with the biggest number of intersections, i.e. the estimated parameters of the calibration marker.

Marker radius value was not fixed, as the magnification factor is not a priori known. This is in contrast to [4] where it was assumed that magnification factor is small and that marker radius can be approximately determined a priori.

\section{B. Approach based on edge detection}

The initial attempt to detect the position of the calibration marker is focused on detecting the strongest circular edge in the image. For that purpose, the gradient of the image was employed, since high intensities of the image gradient magnitude correspond to strong edges in the original image. Fig. 2 shows an example of an unprocessed image obtained by direct detection of radiation, containing human anatomy and a calibration marker, while Fig. 5 shows the gradient magnitude of the example image.

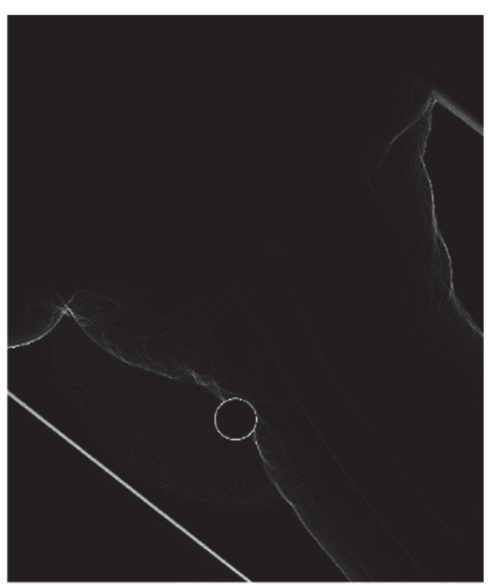

Fig. 5. Gradient magnitudes of image shown in Fig. 2.

The gradient of two-dimensional function $f$ at coordinates $(x, y)$ is defined as the two-dimensional column vector: 


$$
\boldsymbol{\nabla} \boldsymbol{f}=\left[\begin{array}{l}
G_{x} \\
G_{y}
\end{array}\right]=\left[\begin{array}{l}
\frac{\partial f}{\partial x} \\
\frac{\partial f}{\partial y}
\end{array}\right]
$$

The magnitude of this vector is given by:

$$
\nabla f=\operatorname{mag}(\boldsymbol{\nabla} \boldsymbol{f})=\left[G_{x}^{2}+G_{y}^{2}\right]^{1 / 2}
$$

As it is common in practice, the directional gradients were calculated using the Sobel operators, defined as:

$$
S_{x}=\left[\begin{array}{lll}
-1 & 0 & 1 \\
-2 & 0 & 2 \\
-1 & 0 & 1
\end{array}\right] \quad S_{y}=\left[\begin{array}{rrr}
-1 & -2 & -1 \\
0 & 0 & 0 \\
1 & 2 & 1
\end{array}\right]
$$

Edge detection from gradient magnitudes in an image was performed using a threshold obtained by Otsu's method, a technique for determining an optimal global threshold which separates pixels into two classes with maximal inter-class variance [8]. For image with $L$ distinct gray levels, the algorithm assumes that the pixels are divided by a threshold $k$ into classes $C_{0}$ and $C_{1}$. The related class mean levels are:

$$
\begin{gathered}
\mu_{0}=\sum_{i=1}^{k} i \operatorname{Pr}\left(i \mid C_{0}\right)=\sum_{i=1}^{k} i \frac{p_{i}}{\omega_{0}} \\
\mu_{1}=\sum_{i=k+1}^{L} i \operatorname{Pr}\left(i \mid C_{1}\right)=\sum_{i=k+1}^{L} i \frac{p_{i}}{\omega_{1}}
\end{gathered}
$$

and the inter-class variance is defined as:

$$
\sigma_{B}^{2}=\omega_{0} \omega_{1}\left(\mu_{1}-\mu_{0}\right)^{2}
$$

where $i$ denotes pixel intensity, $p_{i}$ is normalized histogram value for intensity $i, \operatorname{Pr}$ is probability operator while $\omega_{0}$ and $\omega_{1}$ are occurrence probabilities of classes $C_{0}$ and $C_{1}$, respectively.

Optimal threshold value $k^{*}$ is selected by maximizing the inter-class variance:

$$
\sigma_{B}^{2}\left(k^{*}\right)=\max _{1<k<L} \sigma_{B}^{2}(k)
$$

A binary image obtained by this method is shown in Fig. 6 .

Pixels detected as edges were allowed to cast votes in the Hough accumulation matrix. A circle with the most votes is considered to represent the calibration marker, as it is supposed to correspond to the most distinct circular shape in the image. An image with a detected calibration marker using this method is shown in Fig. 7.

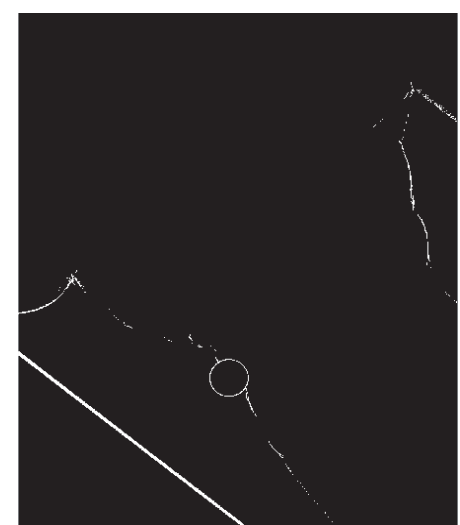

Fig. 6. Binary image obtained by Otsu threshold.

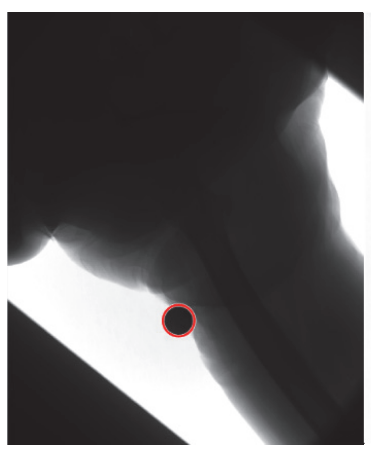

(a)

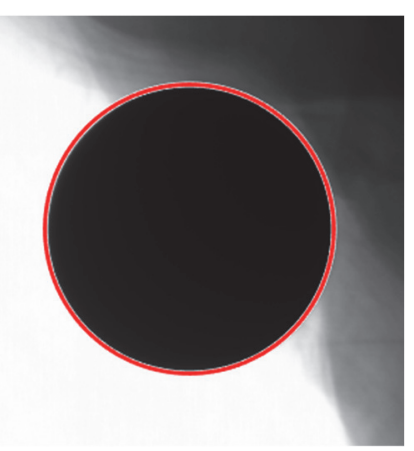

(b)
Fig. 7. Calibration marker detected using Hough transform based on edge detection (a) and magnified calibration marker (b).

\section{Approach without edge detection}

An alternative method for finding the calibration marker is to utilize only the information provided by the gradient magnitudes. Each pixel of the gradient magnitude image is analysed, and instead of incrementing the Hough accumulator cell value by one, accumulator value is increased by the gradient magnitude for the corresponding pixel. At the end of the procedure, the cell with the largest value determines the most distinct circle in the image, considered to represent the calibration marker.

For the sake of processing speed, the original image was reduced 16 times in size using nearest neighbour interpolation, as radiography images can be large in size (around 9 megapixels [9]). An example of calibration marker detection by this method is shown in Fig. 8.

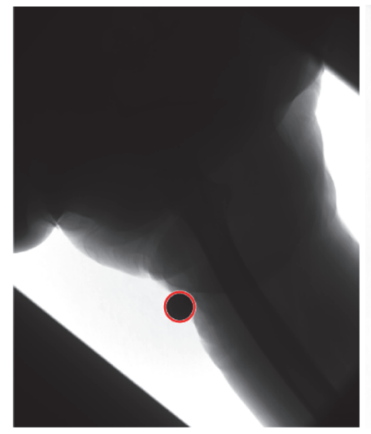

(a)

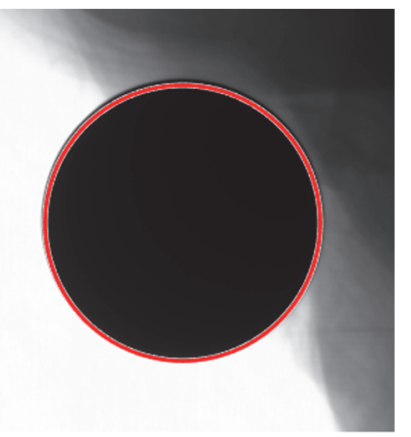

(b)
Fig. 8. Calibration marker detected using Hough transform based on gradient magnitudes (a) and magnified calibration marker (b). 


\section{EVALUATION AND RESULTS}

The evaluation of the two proposed approaches was performed on a dataset consisting of 13 clinical radiography images which contain a calibration marker. The images were obtained during regular clinical routine. Images were obtained using Trixell Pixium 3543 EZ (Trixell, France), with pixel size of $0.148 \mathrm{~mm}$. Used calibration markers have a radius of $25 \mathrm{~mm}$.

For the purpose of evaluation, the positions of markers were also manually annotated in each image, to be compared to the automatically obtained locations. The reference values for centre coordinates and radii of the markers were then calculated as the mean value of manual annotations provided by three trained experts. As the marker size is a priori known, magnification factor was determined using the reference measurements establishing the correspondence of pixel size to the size of the imaged object, enabling conversion of distance in pixels into millimetres.

Both manual annotation and automatic results were compared to the reference measures. As a metric we employed the geometric distance between the centres, the absolute differences of coordinates $x$ and $y$, the absolute differences of radii, as well as the Tanimoto similarity ratio [11], which calculates the spatial matching of two circles.

The mean absolute differences of the results and the reference values are presented in Table 1. The mean absolute distances of reference centres and experimental results are given in Table 2 . The mean values of Tanimoto similarity ratios are given in Table 3 .

TABLE 1: MEAN ABSOLUTE DIFFERENCE OF EXPERIMENTAL RESULTS AND REFERENCE VALUES

\begin{tabular}{l|c|c|c}
\hline $\begin{array}{l}\text { Mean } \\
\text { absolute } \\
\text { difference }\end{array}$ & Manual & $\begin{array}{l}\text { With edge } \\
\text { detection }\end{array}$ & $\begin{array}{c}\text { Without } \\
\text { edge } \\
\text { detection }\end{array}$ \\
\hline$x$-axis & $0.1621 \mathrm{~mm}$ & $0.1276 \mathrm{~mm}$ & $0.3002 \mathrm{~mm}$ \\
\hline$y$-axis & $0.1524 \mathrm{~mm}$ & $0.195 \mathrm{~mm}$ & $0.4694 \mathrm{~mm}$ \\
\hline Radii & $0.1562 \mathrm{~mm}$ & $0.1779 \mathrm{~mm}$ & $0.2352 \mathrm{~mm}$ \\
\hline
\end{tabular}

TABLE 2: MEAN ABSOLUTE DISTANCE OF REFERENCE CENTRES AND EXPERIMENTAL RESULTS

\begin{tabular}{l|l|l|l}
\hline & Manual & $\begin{array}{c}\text { With edge } \\
\text { detection }\end{array}$ & $\begin{array}{c}\text { Without } \\
\text { edge } \\
\text { detection }\end{array}$ \\
\hline $\begin{array}{l}\text { Mean } \\
\text { absolute } \\
\text { distance }\end{array}$ & $0.2506 \mathrm{~mm}$ & $0.2416 \mathrm{~mm}$ & $0.6008 \mathrm{~mm}$ \\
\hline
\end{tabular}

TABLE 3: TANIMOTO SIMILARITY

\begin{tabular}{l|c|c|c}
\hline & Manual & $\begin{array}{c}\text { With edge } \\
\text { detection }\end{array}$ & $\begin{array}{c}\text { Without edge } \\
\text { detection }\end{array}$ \\
\hline $\begin{array}{l}\text { Tanimoto } \\
\text { Similarity }\end{array}$ & $96.46 \%$ & $96.24 \%$ & $93.26 \%$ \\
\hline
\end{tabular}

Upon analysing the presented results, it is clear that the method that uses edge detection is more precise in locating calibration markers in digital radiography images. The advantage of the approach without edge detection is that the threshold for edge detection is not calculated, which makes the approach simpler to implement. A reason for inferior results of the approach without edge detection might be the image size reduction used for processing time reduction (see Section III). Calibration marker parameters were estimated on an image obtained by decreasing the original image size 16 times (both width and height were decreased 4 times), and were afterwards multiplied by 4 to correspond to the original image. This decrease in size resulted in decreased precision of the parameters as they were estimated on an image with reduced resolution. Possibility of attaining better results if the marker radius and centre are adjusted to the full-size image will be addressed in our future work.

Fig. 9 shows images with detected calibration markers using both methods, while Fig. 10 shows magnified calibration markers within those images. The image on the left shows results obtained by the algorithm based on edge detection, while the image on the right shows results obtained by the algorithm based on gradient magnitudes.

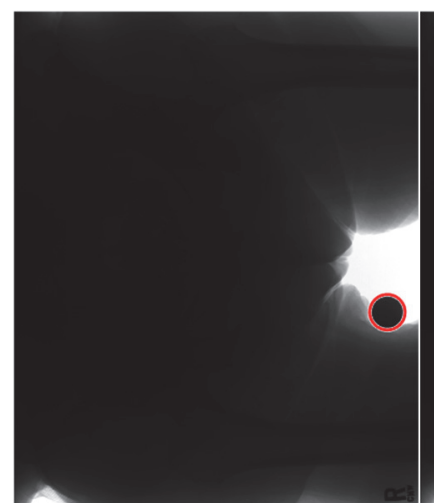

(a)

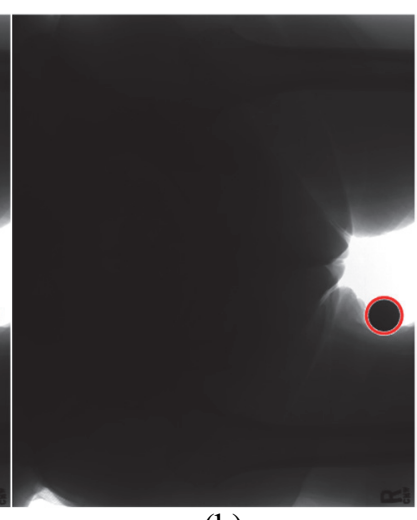

(b)
Fig. 9. Calibration markers detected using Hough transform based on edge detection (a) and using Hough transform based on gradient magnitudes (b).

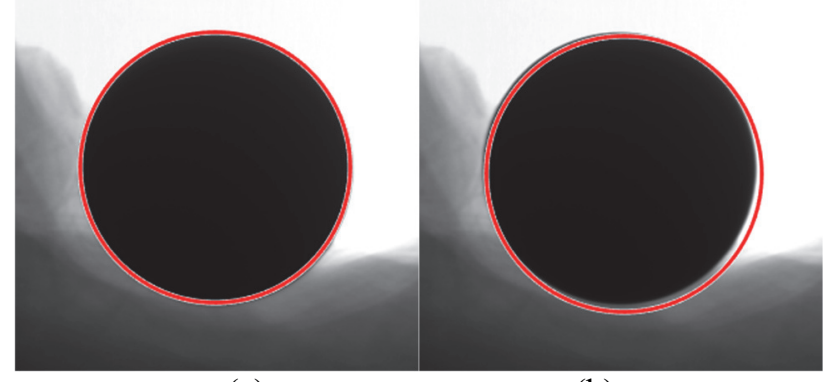

(a)

(b)

Fig. 10. Magnified calibration markers from images shown in Fig. 9a (a) and Fig. 9b (b).

\section{CONCLUSION}

In this paper we analyse two approaches to locating a calibration marker in digital radiography images utilizing the Hough transform. One applies the circular Hough transform on binary images of edges obtained by thresholding image gradient magnitudes. The other approach combines the circular Hough transform with the information provided just by the gradient magnitudes.

By using the approach with binary edge images, we obtain spatial matching with reference circles only $0.22 \%$ less than reference values, whereas by using the approach 
with just gradient magnitudes we obtain spatial matching with reference circles $3.2 \%$ less than reference values.

Inferior results of the approach without edge detection are caused by the image size reduction used to decrease processing time. In our future work we will analyse the possibility of a multi-scale approach. This approach would calculate rough estimates of calibration marker parameters on an image with decreased size, and fine-tune them on the full-size image. Rough estimates obtained on the image with reduced size can be used to determine the approximate neighbourhood of the calibration marker in the full-size image. Parameter refinement can be obtained based on that neighbourhood, disregarding the rest of the image. In this way, the processing time could be reduced while maintaining the parameter estimation precision.

\section{REFERENCES}

[1] B. A. Schueler, "The aapm/rsna physics tutorial for residents general overview of fluoroscopic imaging," RadioGraphics, vol. 20, no. 4, pp. 1115-1126, 2000, pMID: 10903700. [Online]. Available: https://doi.org/10.1148/radiographics.20.4.g00j1301115

[2] B. A. Schueler, "Clinical applications of basic x-ray physics principles." RadioGraphics, vol. 18, no. 3, pp. 731-744, 1998, pMID: 9599394. [Online]. Available: https://doi.org/10.1148/ radiographics.18.3.9599394.

[3] A. R. Cowena, A. G. Daviesa, and M. U. Sivananthan, "The design and imaging characteristics of dynamic, solid-state, flat-panel x-ray image detectors for digital fluoroscopy and fluorography", Clinical Radiology, vol. 63, pp. 1073-1085, 2008.

[4] H. A. Vrooman, E. R. Valstar, G. Brand, D. R. Admiraal, P. M. Rozing, J. H.C Reiber, "Fast and accurate automated measurements in digitized stereophotogrammetric radiographs," Journal of Biomechanics, vol. 31, no. 5, pp. 491-498, 1998. [Online]. Available: https://doi.org/10.1016/S0021-9290(98)00025-6.

[5] C. K. Boese, P. Lechler, L. Rose, J. Dargel, J. Oppermann, P. Eysel, H. Geiges, J. Bredow, "Calibration Markers for Digital Templating in Total Hip Arthroplasty," PLOS ONE, vol. 10, no. 7, pp. 1-12, 2015, doi: 10.1371/journal.pone.0128529.

[6] R. O. Duda, P. E. Hart, "Use of the Hough Transformation to detect lines and curves in pictures," Comm. ACM, vol. 15, no. 1, pp. 11-15, 1972, doi: 10.1145/361237.361242.

[7] L. Novović, V. Ostojić, Đ. Starčević, V. Petrović, "Automatic calibration marker detection for radiography images", 2018 26th Telecommunications forum (TELFOR), Belgrade, 2018, pp. 309-312.

[8] N. Otsu, "A Threshold Selection Method from Gray-Level Histograms," in IEEE Trans. Syst., Man, Cybern., vol. 9, no. 1, pp. 62-66, Jan. 1979, doi: 10.1109/TSMC.1979.4310076.

[9] E. L. Nickoloff, "Aapm/rsna physics tutorial for residents: Physics of flat-panel fluoroscopy systems," RadioGraphics, vol. 31, no. 2, pp. 591-602, 2011. [Online]. Available: http://dx.doi.org/10.1148/rg.312105185.

[10] T. T. Tanimoto, "An elementary mathematical theory of classification and prediction," Internal IBM Technical Report, 1958.

[11] D. J. Rogers, T. T. Tanimoto, "A Computer Program for Classifying Plants," Science, vol. 132, no 3434, pp. 1115-1118, 1960, doi: 10.1126/science.132.3434.1115. 